\title{
A versatile plasmid system for reconstitution and analysis of mammalian ubiquitination cascades in yeast
}

\author{
Rossella Avagliano Trezza ${ }^{1, \#}$, Janny van den Burg ${ }^{1}$, Nico van den Oever ${ }^{1}$ and Ben Distel ${ }^{1,2, *}$ \\ ${ }^{1}$ Department of Medical Biochemistry, Academic Medical Center, University of Amsterdam, Amsterdam, The Netherlands. \\ 2 Department of Neuroscience, Erasmus Medical Center, Rotterdam, The Netherlands. \\ \# Current Address: Department of Neuroscience, Erasmus Medical Center, Rotterdam, The Netherlands. \\ * Corresponding Author: \\ Ben Distel, PhD, Academic Medical Center, University of Amsterdam, Department of Medical Biochemistry; ErasmusMC, Department \\ of Neuroscience, Wijtemaweg 80, 3015 CN Rotterdam; Phone: (+31) 010 7043571; E-mail: b.distel@amc.uva.nl
}

\begin{abstract}
Ubiquitination is a posttranslational protein modification that regulates most aspects of cellular life. The sheer number of ubiquitination enzymes that are present in a mammalian cell, over $\mathbf{7 0 0}$ in total, has thus far hampered the analysis of distinct protein ubiquitination cascades in a cellular context. To overcome this complexity we have developed a versatile vector system that allows the reconstitution of specific ubiquitination cascades in the model eukaryote Saccharomyces cerevisae (baker's yeast). The vector system consists of $\mathbf{3 2}$ modular yeast shuttle plasmids allowing inducible or constitutive expression of up to four proteins of interest in a single cell. To demonstrate the validity of the system, we show that co-expression in yeast of the mammalian HECT type E3 ubiquitin ligase E6AP (E6-Associated Protein) and a model substrate faithfully recapitulates E6AP-dependent substrate ubiquitination and degradation. In addition, we show that the endogenous sumoylation pathway of $S$. cerevisiae can specifically sumoylate mouse PML (Promyelocytic leukemia protein). In conclusion, the yeast vector system described in this paper provides a versatile tool to study complex posttranslational modifications in a cellular setting.
\end{abstract}

doi: $10.15698 /$ mic2018.03.620

Received originally: 03.07.2017;

in revised form: 23.11.2017,

Accepted 27.11.2017,

Published 05.12.2017.

Keywords: ubiquitination, sumoylation, modular vectors, inducible expression, yeast, Saccharomyces cerevisiae.
Abbreviations:
CTA - catalase $A$,
$\mathrm{CHX}$ - cycloheximide,
E6AP-E6-associated protein,
HECT - homologous to E6AP C-
terminus,
MCS - multiple cloning site,
$P M L$ - promyelocytic leukemia
protein,
SUMO - small ubiquitin-like modifier, UAS - upstream activating sequence, Ub-ubiquitin.

\section{INTRODUCTION}

The process of ubiquitination, involving the covalent conjugation of the small protein ubiquitin (Ub) to a substrate protein, is one of the most finely tuned mechanisms of post-translational modification in eukaryotic biology [1]. Ubiquitination is accomplished through an elaborate enzymatic cascade involving the concerted action of three distinct types of enzymes: E1 Ub-activating, E2 Ubconjugating and $\mathrm{E} 3 \mathrm{Ub}$ ligase. First, $\mathrm{Ub}$ is activated by one of two cellular E1 enzymes, then transferred to $40 \mathrm{E2}$ enzymes that interact with more than 600 E3 ligases. The substrate specificity is determined in the last step of the cascade, catalysed by the E3, in which Ub is covalently attached to a lysine residue of a specific target protein [2]. The two main groups of E3 ligases are HECT (Homologous to E6-AP C-terminus) and RING (Really interesting new gene) ligases. The HECT E3s contain a conserved active site cysteine that acts as an acceptor for Ub from E2 conjugating enzymes. The $\mathrm{Ub}$ is then transferred onto a Lysine residue of the target protein. RING E3s ligases on the other hand do not have an active site cysteine, but function as a scaffold that enhances the transfer of Ub from the E2 active site directly onto the substrate [3].

$\mathrm{Ub}$ itself contains seven lysine residues and a $\mathrm{N}$ terminal $\mathrm{NH}_{2}$ group, each of which can serve as a substrate of ubiquitination, resulting in the formation of $\mathrm{Ub}$ chains consisting of multiple $\mathrm{Ub}$ moieties. This ability of $\mathrm{Ub}$ to form chains of at least eight different linkages adds to the complexity of the ubiquitination system. Indeed, the fate of the ubiquitinated substrate is, to a large extent, determined by the length and type of linkage between Ub moieties [4]. For example, chains linked via Lys63 (K63) are im- 
plicated in DNA repair, signaling and endocytosis [5]. On the other hand, substrates that receive poly-ubiquitin chains linked via Lys48 (K48) or Lys11 (K11) are targeted for degradation by the $26 \mathrm{~S}$ proteasome $[6,7]$, a multi-protein complex responsible for proteolysis and ubiquitin recycling. The $26 \mathrm{~S}$ proteasome is a conserved proteolytic machinery present in both the nucleus and the cytosol of eukaryotic cells [8]. Proteasomes are also present in some bacterial species where they are involved in the degradation of pupylated proteins [9], a type of post-translational modification that is functionally analogous to ubiquitination, but chemically distinct [10].

The analysis of specific ubiquitination cascades in a cellular setting is a major challenge given that mammalian cells express more than 700 ubiquitination enzymes, which often have overlapping substrate specificities $[11,12]$. To reduce complexity, cell-free approaches have been developed that utilize reconstituted ubiquitination cascades. However, these cell-free assays require extensive protein purification of the respective ubiquitination enzymes as well as their substrate. A bacterial ubiquitination system has recently been developed that allows the analysis of specific ubiquitination cascades in a cellular context [13]. The intrinsic differences of proteasomes in bacteria [9], however, limit the analysis of ubiquitinated substrates. To overcome these limitations we have established a cellular system for reconstitution of mammalian E3 ubiquitin ligase-dependent substrate modification and degradation. This system employs the model eukaryote Saccharomyces cerevisiae (baker's yeast) as a host for co-expression of E2, E3 and substrate of a specific mammalian ubiquitination cascade, while the yeast cell provides the ubiquitin and the E1 enzyme. Yeast shuttle plasmids were developed that allow expression and detection of up to four proteins of interest in a single yeast cell. Gene expression is regulated by three different types of promoters: galactose inducible GAL1 promoter (available in three different strengths), the $\mathrm{CuSO}_{4}$ inducible CUP promoter or the strong constitutive TEF promoter. To improve stability and detection of polyubiquitinated targets, a strain was constructed that lacks the $A B C$ transporter $P D R 5$ rendering the cells more susceptible to the proteasome inhibitor MG132 [14].

Here we demonstrate the validity of our system by showing specific ubiquitination and proteasomal degradation of an established target of E6AP, a HECT type E3 ligase that is deficient in patients with Angelman syndrome [15, 16]. In addition, we demonstrate that the system can also be used to study other types of post-translational modifications by showing sumoylation of the mouse PML protein in yeast.

\section{RESULTS AND DISCUSSION \\ Plasmid construction}

To enable study of mammalian ubiquitination cascades in yeast we constructed a modular set of plasmids (called pRA) that allow for: a) inducible or constitutive expression of the enzymes of the ubiquitination cascade and substrates, and b) detection of the gene products bearing $\mathrm{N}$ -

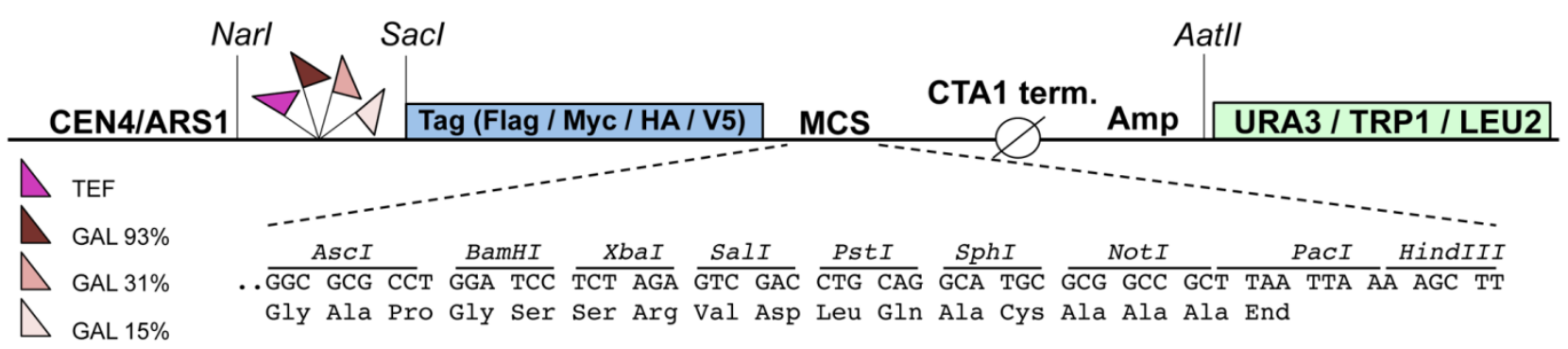

B

\begin{tabular}{|c|c|c|c|c|c|}
\hline $2 \mu \mathrm{m}$ & Amp & \begin{tabular}{|l|} 
Tag (6His-Myc) \\
\end{tabular} & Ub/Smt3 & Cycie & HIS3 \\
\hline
\end{tabular}

FIGURE 1: Schematic representation of the constructed vectors. The pRA vectors (A) are yeast centromeric shuttle plasmids that carry an auxotrophic marker (URA3, TRP1 or LEU2 gene) for selection in yeast and the ampicillin (Amp) resistance gene for selection in E. coli. The gene of interest can be cloned into the multiple cloning site (MCS) under control of either the inducible GAL1 promoter (3 different strengths) or the constitutive TEF promoter. The expressed protein will bear any of the four (Flag, Myc, HA or V5) N-terminal epitope tags allowing easy detection. All restriction sites indicated are unique. (B) High copy $(2 \mu \mathrm{m})$ yeast shuttle plasmids carrying the HIS3 auxotrophic marker and expressing either 6xHis-Myc tagged ubiquitin (Ub) or SUMO (Smt3) under control of the copper inducible promoter (CUP). CTA1 and Cyc1 term (inator), transcription termination sequences derived from the yeast Catalase A and Cytochrome $\mathrm{c} 1$ gene, respectively. 
terminal epitope tags. The plasmid backbone for the vector system is derived from the YCplac series of single copy yeast shuttle plasmids that carry the URA3, TRP1 or LEU2 auxotrophic selection markers [17]. To induce expression of the gene of interest we made use of the tightly controlled GAL1 promoter [18]. The GAL1 promoter is actively repressed by glucose and changing the carbon source from glucose to galactose induces protein expression. The use of an inducible promoter can facilitate the expression of toxic proteins [19]. The GAL1 promoter exists in three different versions, "strong" (93\% activity, contains four Gal4p U $p$ stream Activating Sequences [UAS], "medium" (31\% activity, three Gal4p UASs) and "weak" (15\% activity, two Gal4p UASs), allowing further fine-tuning of gene expression levels. In order to facilitate constitutive expression we employed the strong TEF promoter [20]. To enable protein detection four distinct $\mathrm{N}$-terminal epitope tags, HA, V5, Flag and Myc were inserted in each plasmid. Finally, each plasmid harbours a new multiple cloning site (MCS) with unique restriction sites for facile cloning of the gene of interest. The pRA vector system currently consists of 32 plasmids each of which harbours a different combination of promoter, tag and auxotrophic marker (see also Table S1), but this set can be extended up to 48 by including all available GAL1 promoter variants (Fig 1). The advantage of pRA vector system compared to previously published collections of yeast expression vectors $[21,22,23]$ is that different $\mathrm{N}$-terminal epitope tags are included in the vector allowing simultaneous expression and detection of multiple genes of interest in a single yeast cell. To facilitate the detection and purification of ubiquitinated (or sumoylated) substrates two separate high copy $(2 \mu \mathrm{m})$ plasmids were constructed that express either 6xHis-Myc tagged ubiquitin or 6xHis-Myc tagged SUMO (Smt3) under the control of the copper inducible promoter CUP1 [24]. These two plasmids harbor the HIS3 auxotrophic marker and each can be combined with up to three pRA plasmids, bringing the total number of genes expressed in a single yeast cell to four.

\section{Galactose inducible expression}

To test the functionality of the GAL1 promoter plasmids we cloned E6-E7, one of the canonical targets of the HECT E3 ligase E6AP, in three different vectors harboring the $93 \%-$,
31\%- and 15\%-GAL1 promoter, respectively (see Material and methods). The E6-E7 fusion protein consists of the entire human papillomavirus (HPV)-16 E7 fused to HPV-16 E6 [25]. E6-E7 was tagged at its N-terminus with the Flag tag. Constructs were transformed into yeast yRA2, a derivative of $S$. cerevisiae strain BY4741 in which the TRP1 gene was knocked out (see Material and methods). Transformants were first cultured in medium containing $2 \%$ glucose, then shifted to a medium with $2 \%$ raffinose and $0.1 \%$ glucose, and finally inoculated in medium containing $2 \%$ galactose to induce expression of E6-E7 for the indicated time points. As shown in Figure 2, E6-E7 was undetectable in total lysates of cells grown in $2 \%$ raffinose/0.1\% glucose medium $(t=0)$, indicating tight glucose repression of E6-E7 expression. Within $30 \mathrm{~min}$ after the shift to galactosecontaining medium E6-E7 could be detected in cells expressing the gene from the strongest version of the GAL1 promoter, the 93\%-GAL1. All three promoter-versions showed a gradual increase of E6-E7 expression with time, reaching the highest levels with the 93\%-GAL1 promoter. Taken together, these data show that by using different versions of the GAL1 promoter protein expressions levels can be modulated.

\section{E6AP-dependent ubiquitination of E6-E7}

Previous work has shown that the E6-E7 fusion protein is efficiently ubiquitinated by E6AP in a cell-free system [25]. We therefore set out to determine whether E6AP is capable of specifically ubiquitinating the E6-E7 fusion protein in the heterologous yeast system. To reconstitute this specific ubiquitination cascade in yeast, mouse E6AP (wild-type and the catalytically inactive mutant, E6AP-C817S) and its cognate $\mathrm{E} 2$, mouse $\mathrm{UBCH} 7$, were cloned under control of the constitutive TEF promoter, each bearing different tags (HA for E6AP and Flag for UBCH7). The yRA2 strain, in which the plasma-membrane $A B C$ transporter Pdr5 was deleted by homologous recombination, was cotransformed with E6-E7 (V5 tag, 93\%-GAL1 promoter), E6AP (wt or mutant) and either UBCH7 or an empty plasmid. Transformants were pre-cultured on $2 \%$ raffinose $/ 0.1 \%$ glucose and shifted to $2 \%$ galactose to induce the expression of E6-E7. After 1 hour of galactose induction, $75 \mu \mathrm{M}$ MG132 was added to inhibit proteasomal activity

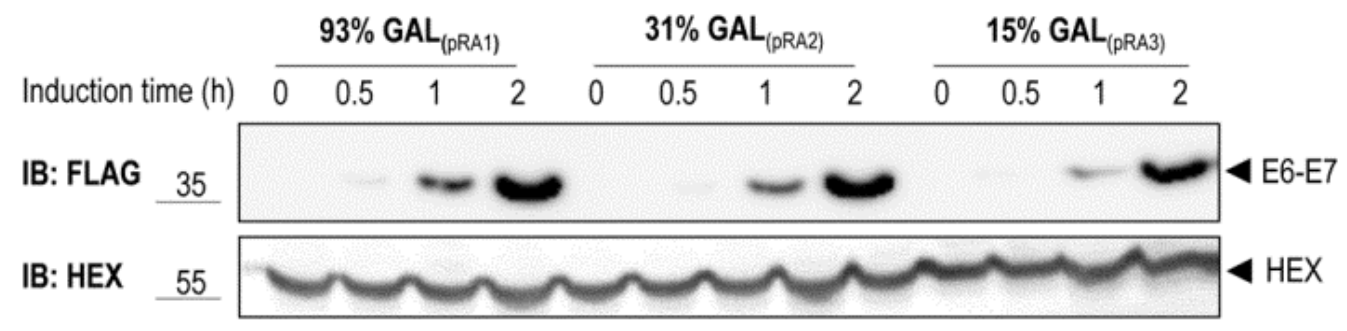

FIGURE 2: Galactose inducible expression of E6-E7. Yeast strain yRA2 transformed with E6-E7 cloned in pRA1 (Flag tag, URA3 marker, 93\%GAL1 promoter), pRA2 (Flag tag, URA3 marker, 31\%-GAL1 promoter) and pRA3 (Flag tag, URA3 marker, 15\%-GAL1 promoter), was induced for the indicated time points by the addition of $2 \%$ galactose. Cells were lysed and equivalent amounts of protein extract were analyzed by SDSPAGE and anti-Flag immunoblotting. Hexokinase is used as a loading control. 
and allow accumulation of ubiquitinated proteins [26]. Immunoblot analysis of yeast total lysates revealed that, in addition to the band corresponding to the unmodified E6E7 fusion protein, slower migrating bands accumulated in cells expressing wild type E6AP (Figure $3 \mathrm{~A}$ ). The fact that these bands are absent in cells co-expressing the catalytically inactive E6AP (E6AP-C817S) suggests that E6-E7 can be ubiquitinated in yeast in an E6AP-dependent manner. To unambiguously show that these slower migrating species represent (poly)-ubiquitinated forms of E6-E7 the experiment was repeated with cells co-transformed with 6xHis-Myc-tagged ubiquitin (6xHis-Myc-Ub, driven by the CUP1 promoter) or empty plasmid. Efficient conjugation of $6 \mathrm{xHis}-\mathrm{Myc}-\mathrm{Ub}$ to proteins was verified by anti-myc immunoblotting (Figure S1). Conjugation of the recombinant 6xHis-Myc-Ub to a protein will result in a slower electrophoretic mobility of the ubiquitinated protein compared to a protein modified with wild-type (endogenous) ubiquitin due to the addition of the $6 \mathrm{xHis}-\mathrm{Myc}$ tag on ubiquitin. As shown in Figure 3B (right panel) the slower migrating E6-E7 bands observed in cells expressing the catalytically active E6AP shifted to an apparent higher molecular weight upon overexpression of $6 \mathrm{xHis-Myc-Ub.} \mathrm{This} \mathrm{data} \mathrm{confirm} \mathrm{that}$ these slower migrating bands are indeed the result of E6AP-dependent ubiquitination of E6-E7. Notably, in the absence of $\mathrm{UBCH} 7$, similar levels of E6-E7 ubiquitination were observed indicating that one of the endogenous yeast E2 enzymes [27] can functionally interact with E6AP to ubiquitinate its target (Figure S2). This data support the notion that for the analysis of a specific mammalian ubiquitination cascade in yeast, expression of the E3 ligase and its target may be sufficent, a major advantage in cases where not all components of the specific ubiquitination cascade are known.

\section{Degradation of E6-E7}

To test if E6AP-dependent poly-ubiquitination of E6-E7 leads to proteasome-mediated degradation of the fusion protein in the reconstituted yeast system, we performed a cycloheximide $(\mathrm{CHX})$ chase experiment. Yeast cells were
A

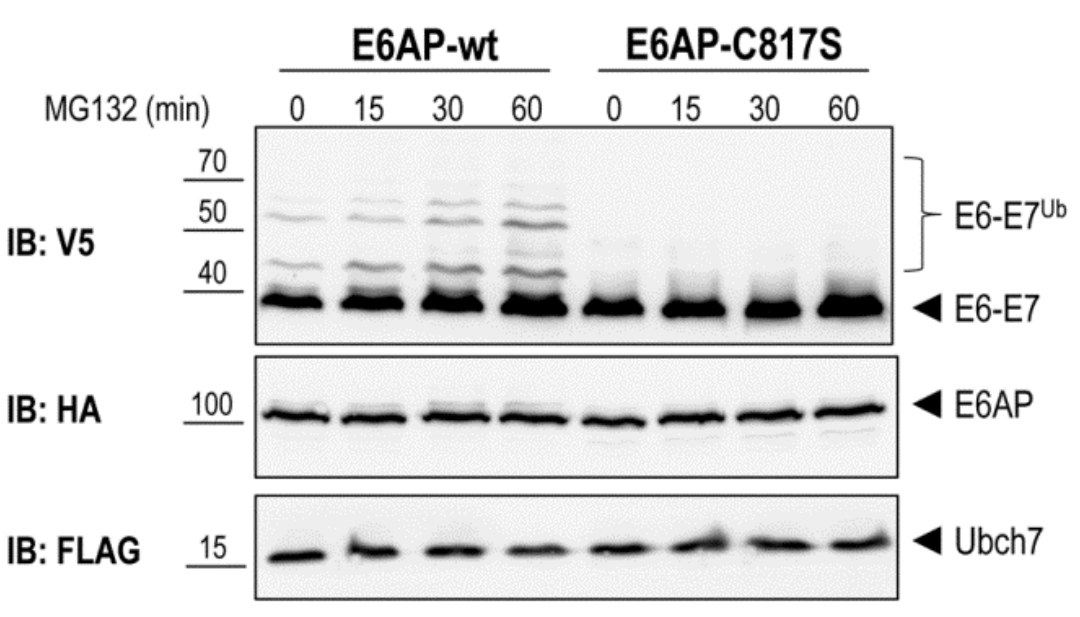

B
FIGURE 3: E6AP-dependent ubiquitination of E6-E7. (A) Yeast strain yRA2 was transformed with E6AP (wild type or catalytically inactive mutant) cloned in pRA306 (HA tag, LEU2 marker, TEF promoter), UBCH7 cloned in pRA300 (Flag tag, URA3 marker, TEF promoter) and E6-E7 cloned in pRA20 (V5 tag, TRP1 marker, 93\%-GAL1 promoter). Following 1 hour of galactose induction, cells were treated with $75 \mu \mathrm{M}$ MG132 for the indicated time points. Cells were lysed and equivalent amounts of protein extract were analyzed by SDS-PAGE and immunoblotting using the indicated antibodies. (B) Yeast strain YRA2 was transformed with EGAP (wild type or catalytically inactive mutant) cloned in pRA306 (HA tag, LEU2 marker, TEF promoter), E6E7 cloned in pRA20 (V5 tag, TRP1 marker, 93\%-GAL1 promoter) and 6xHis-Myc-Ub (Myc tag, HIS3 marker, CUP promoter) or empty p423 vector. Expression of $6 x \mathrm{His}-\mathrm{Myc}-\mathrm{Ub}$ was induced by addition of $\mathrm{CuSO}_{4}(100 \mu \mathrm{M})$. Following 1 hour of galactose induction, cells were treated with $50 \mu \mathrm{M}$ MG132 for 120 min. Cells were lysed and equivalent amounts of protein extract were analyzed by SDS-PAGE and immunoblotting using anti-V5 antibody. The band marked with an asterisk $\left({ }^{*}\right)$ is an example of an ubiquitinated E6-E7 band that in cells overexpressing 6xHis-Myc-Ub, generates additionally slower migrating species (arrow head) due to the incorporation of the 6xHis-Myc-tagged ubiquitin. 
co-transformed with E6-E7, UBCH7, and E6AP-wt or E6APC817S. After galactose induction, protein synthesis was inhibited by treating the cells with $50 \mu \mathrm{g} / \mathrm{ml} \mathrm{CHX}$ for the indicated times (Figure 4). In cells expressing E6AP-wt, E6E7 rapidly disappeared upon addition of $\mathrm{CHX}$. In contrast, no appreciable reduction of E6-E7 levels was observed in cells co-transformed with the catalytically inactive E6AP (E6AP-C817S). These data indicate that E6AP-catalyzed ubiquitination of E6-E7 in yeast results in rapid protein degradation, a process most likely mediated by the $26 \mathrm{~S}$ proteasome. Together, we show that a complex cellular mechanism such as the ubiquitination and degradation of a substrate via the ubiquitin proteasome system can be reconstituted in yeast by expressing only the specific E3 and its substrate. Such an approach is only possible in a model eukaryotic setting, such as yeast, where biological mechanisms are highly conserved.

\section{Sumoylation of promyelocytic leukemia protein}

Another example of a biological process that is conserved between mammals and yeast is sumoylation, a posttranslational modification very similar to ubiquitination [28]. Sumoylation is the process by which proteins are covalently tagged with a SUMO (ㅍmall ubiquitin- like modifier) moiety that shares $\sim 20 \%$ similarity with Ub. SUMO proteins are highly conserved from yeast to mammals. In yeast, only one SUMO protein (Smt3) exists, while mammalian cells express three major SUMO isoforms (SUMO-1, SUMO2 , and SUMO-3). Sumoylation, the linkage of SUMO to a target protein through an isopeptide bond, is catalyzed by a series of enzymatic reactions similar to that of ubiquitination. First, SUMO/Smt3 is activated by an E1 SUMOactivating enzyme (SAE1/ 2 in mammals and UBA2 in yeast), which is followed by transfer of the activated SUMO/Smt3 to a SUMO-specific E2, Ubc9. From here, either the SU$\mathrm{MO} / \mathrm{Smt3}$ is directly transferred to a lysine side-chain of the target protein or the transfer is indirectly and requires an SUMO E3 ligase. In contrast to ubiquitination, sumoylation often occurs on a consensus motif, $\Psi$-K-x-D/E ( $\Psi$; hydrophobic residue, $\mathrm{K}$; lysine, $\mathrm{x}$; any amino acid and $\mathrm{D} / \mathrm{E}$; aspartic acid/glutamic acid) [28]. The PML protein offers a well-established example of a protein that is sumoylated. PML is the key player in the generation of Nuclear Bodies
(NB) involved in a myriad of processes, including cellular senescence, virus response, and apoptosis [29]. PML contains three sumoylation consensus motifs each of which are targeted by sumoylation. Sumoylation of these consensus lysines is essential for the recruitment of nuclear proteins to the NB [30, 31].

To test if PML sumoylation can be recapitulated in yeast we overexpressed V5-tagged mouse PML under the control of the 93\%-GAL1 promoter in presence or absence of 6xHis-Myc-Smt3 driven by the CUP1 promoter. As shown in Figure $5 \mathrm{~A}$, following galactose-induction and in the absence of $6 \mathrm{xHis-Myc-Smt3}$ up to three slower migrating bands appeared above the main band representing PML, suggesting targeting of all three consensus-sumoylation sites in PML. In cells overexpressing 6xHis-Myc-Smt3 these slower migrating bands were split up in two or more bands indicating successful incorporation of recombinant 6xHis-MycSmt3 and supporting the notion that these slower migrating species represent sumoylated forms of PML. Similar results were obtained when human PML was expressed in yeast (data not shown). This data strongly suggest that sumoylation of mouse PML can be faithfully reproduced in yeast by employing its endogenous sumoylation machinery. In line with previous observations [32] overexpression of PML resulted in an overall increase of Smt3-conjugated species, most likely representing Smt3 linked to PML and to endogenous yeast proteins (Fig. 5B). How PML stimulates $\mathrm{Smt} 3$ conjugation in yeast remains to be determined.

In conclusion, we show that the use of a model eukaryote, in combination with a highly versatile plasmid system that allows regulated expression of multiple heterologous proteins, can facilitate the analysis of complex (mammalian) post-translational modification cascades such as ubiquitination and sumoylation in a cellular context.

\section{MATERIALS AND METHODS \\ Plasmid generation}

The vector backbone of the pRA plasmids is pEL43 [33], an YCplac33 derived yeast shuttle plasmid [17] harboring the CTA (Catalase A) promoter and CTA terminator separated by a multiple cloning site (MCS), the URA3 auxotrophic marker and the ARS1/CEN4 region for autonomous replication in yeast. The three different GAL1 promoters (93\%, 31\% and $15 \%$ ) were

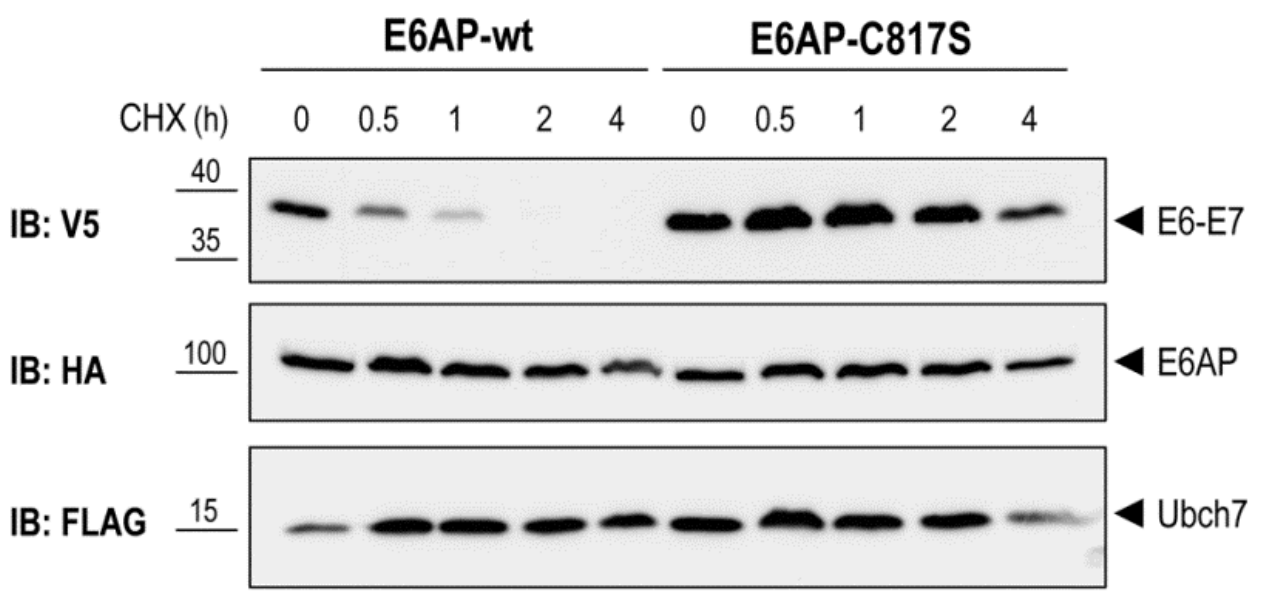

FIGURE 4: E6AP-dependent degradation of E6-E7. Yeast strain YRA2 was transformed with E6AP, UBCH7 and E6-E7 as described in the legend to Figure 3. Following 1 hour of galactose induction, cells were treated with $50 \mu \mathrm{g} / \mathrm{ml} \mathrm{CHX}$ for the indicated time points. Cells were lysed and equivalent amounts of protein extract were analyzed by SDSPAGE and immunoblotting using the indicated antibodies. 
obtained by PCR on yeast genomic DNA using primers containing a 5' Narl and a 3' Sacl site, respectively (Table S2) and subsequently cloned in PEL43 digested with Narl and Sacl, thereby replacing the CTA promoter with the GAL1 promoter sequences. Next, a new MCS was inserted in each of the three vectors between the Pstl and HindIII sites using double stranded (ds) oligonucleotides (Table S2). At this point, ds oligonucleotides encoding the four different $\mathrm{N}$-terminal epitope tags (HA, V5, Flag and Myc) followed by an Ascl site were inserted between the Sacl and BamHI restriction sites of each plasmid. Finally, the URA3 auxotrophic marker in these plasmids was replaced by TRP1 or LEU2 by cloning the NarlAatll fragments encompassing promoter, tag, MCS and CTA terminator into YCplac22 (TRP1) and Ycplac111 (LEU2) digested with Narl and AatIl. The TEF promoter was obtained by PCR from pTEF-URA [34] (generous gift of Jens Nilsen) using primers containing a $5^{\prime} \mathrm{Narl}$ and a 3' Sacl and subsequently cloned in pRA1, 4, 7, 10 and 13-20, digested with Narl and Sacl, thereby replacing the 93\%-GAL1 promoter with the TEF promoter sequence. Ubch7 (Ube2I3) was amplified by PCR from image clone BC106149 (clone ID 4502101) using primers containing a 5' Ascl and a 3' Sall site, respectively (Table S2) and subsequently cloned in pRA300 digested with Ascl and Sall. To obtain a higher expression of the E2 enzyme, the CEN/ARS1 sequence in pRA58 was replaced by the $2 \mu \mathrm{m}$ origin of replication derived from YEplac195, resulting in plasmid pRA60. Ube $3 a$ was amplified by PCR with primers p881 and p882 from mouse brain cDNA (generous gift of Ype Elgersma) and subsequently cloned in pRA306. Pml was amplified by PCR from image clone BC020990 (clone ID 4188386) using primers containing a 5' Ascl and a 3' Sall site, respectively (Table S2) and subsequently cloned in PRA20. E6-E7 fusion protein was amplified by fusion PCR (to delete the internal Sall site) from plasmid \#95 [25] (generous gift of Jon Huibregtse) using external primers containing a $5^{\prime}$ BamHI and a $3^{\prime}$ Sall site, respectively (S2 Table) and subsequently cloned in pRA20, generating pJB322. The BamHI-Notl fragment encompassing E6-E7 was isolated from pJB322 and cloned in plasmids pRA1, pRA2 and pRA3 digested with BamHI andNotl.

6xHis-Myc-Ub and 6xHis-Myc-Smt3 plasmids are based on p423, a multi-copy $(2 \mu \mathrm{m})$ yeast shuttle plasmid harboring the MET25 promoter, Cyc1 terminator and HIS3 auxotrophic marker [23]. A three fragment ligation was used to construct 6xHis-Myc-Ub as follows: the EcoRI-Mlul fragment from pCW129 [35] encompassing 6xHis-Myc-Ub and part of the Cyc1 terminator sequences and the Sacl-EcoRI fragment from pES7 (generous gift of Mike Ellison, University of Alberta) harboring the CUP1 promoter were ligated into p423 digested with Sacl and Mlul, generating 6xHis-Myc-Ub (pBD238, Table S2). To construct $6 x$ His-Myc-Smt3, the Smt3 gene was amplified from yeast genomic DNA, thereby introducing a $5^{\prime} \mathrm{BamHI}$ site and a $3^{\prime} K p n l$ site. The BamHI-Kpnl fragment was subsequently cloned into pCW129 digested with Bg/ll and Kpnl, thereby replacing the Ub gene with Smt3. The BamHI-Mlul fragment of the resulting plasmid, encompassing the CUP promoter, 6xHis-Myc-Smt3 and part of the Cyc1 terminator sequences, was isolated and cloned into pBD238 cut with BamHI and Mlul, generating 6xHis-Myc-Smt3 (pNO3, S1 Table). A complete list of plasmids generated in this study can be found in Table S1. Plasmids will be deposited at Addgene.

\section{Yeast strains}

Strain yRA1 was derived from S. cerevisiae strain BY4741 (MATa his3 $\Delta 1$ leu2 $\Delta 0$ met15 $\Delta 0$ ura3 $\Delta O$ ) by partial deletion of the $T R P 1$ gene ( $0.51 \mathrm{~kb}$ deletion) using the loxP/Cre gene disruption and marker rescue procedure as previously described [36]. This deletion keeps elements of the UAS (upstream activating sequence) required for expression of the adjacent GAL3 gene intact thereby not affecting the kinetics of galactose induction [37]. The loxP/Cre system was also used to delete the $A B C$ transporter gene PDR5 in yRA1 thereby generating yRA2.
A

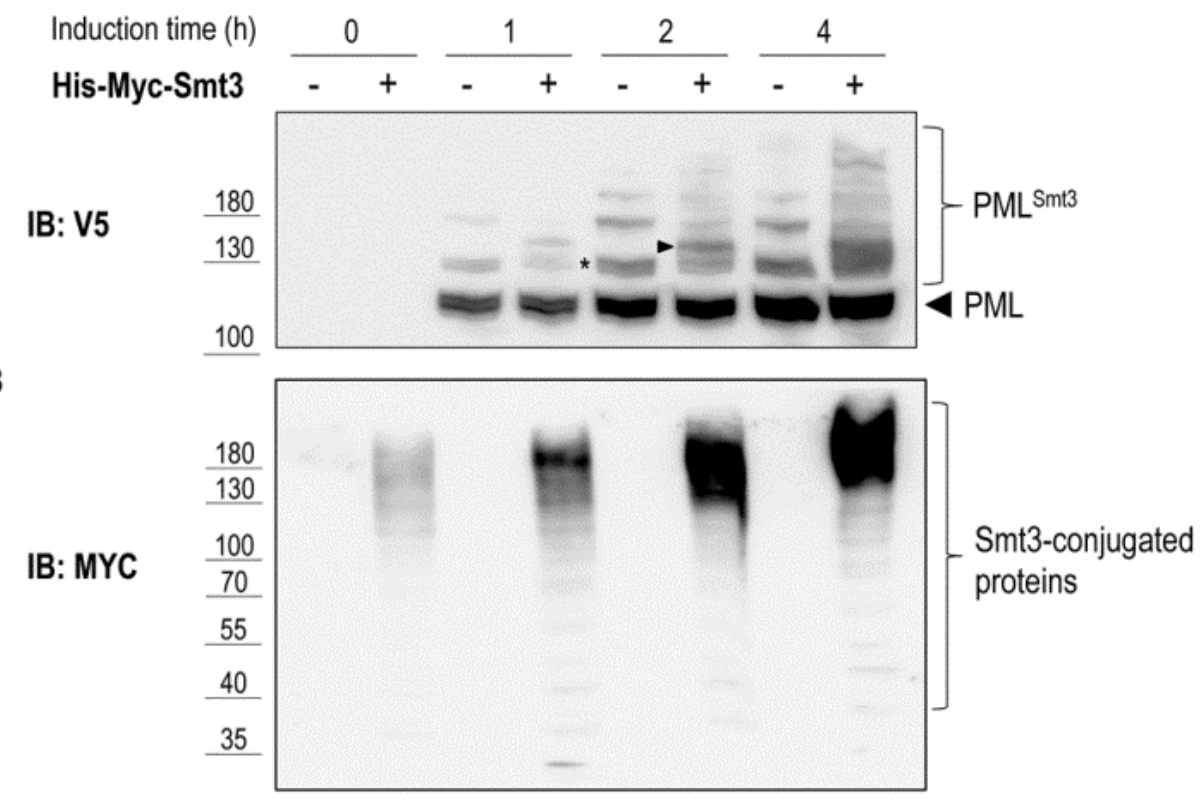

FIGURE 5: Sumoylation of mouse PML in yeast. Yeast strain YRA1 was transformed with PML cloned in pRA20 (V5 tag, TRP1 marker, 93\%-GAL1 promoter) and with a plasmid expressing 6xHis-Myc-Smt3 under control of the CUP promoter (HIS3 marker) or with empty vector. Expression of PML and Smt3 was induced by addition of galactose $(2 \%[\mathrm{w} / \mathrm{v}])$ and $\mathrm{CuSO}_{4}(100 \mu \mathrm{M})$, respectively. Cells were lysed and equivalent amounts of protein extract were analyzed by SDS-PAGE and anti-V5 (A) or anti-Myc (B) immunoblotting. The band marked with an asterisk $\left({ }^{*}\right)$ in panel $A$ is an example of PML modified by a single Smt3 moiety that, in cells overexpressing $6 x \mathrm{His}-\mathrm{myc}-\mathrm{Smt} 3$, gives rise to a slower migrating species (arrow head) due to covalent attachment of the 6xhis-Myc-tagged Smt3. 


\section{Yeast transformation}

Transformation of S. cerevisiae cells was conducted as previously described using the lithium-acetate protocol [38].

\section{Galactose induction}

On day 1 , yeast colonies transformed with the desired plasmid(s) were inoculated in $3 \mathrm{ml}$ of minimal medium (2\% [w/v] glucose, $0.67 \%[\mathrm{w} / \mathrm{v}]$ Yeast Nitrogen Base [DIFCO] and amino acids as required) and incubated over night $(\mathrm{O} / \mathrm{N})$ at $28^{\circ} \mathrm{C}$ while shaking at $200 \mathrm{rpm}$.

On day 2 , cells of the $\mathrm{O} / \mathrm{N}$ culture were re-inoculated in 10 $\mathrm{ml}$ of minimal medium at an $\mathrm{OD}_{600}$ of 0.2 and grown until they reached an $\mathrm{OD}_{600}$ of 0.8 (approx. 7-8h). Upon reaching the desired OD value, cells were re-inoculated in $10 \mathrm{ml}$ of raffinose minimal medium $(2 \%[\mathrm{w} / \mathrm{v}]$ raffinose, $0.1 \%[\mathrm{w} / \mathrm{v}]$ glucose, $0.67 \%[\mathrm{w} / \mathrm{v}$ ] Yeast Nitrogen Base [DIFCO] and amino acids as required) at a starting $\mathrm{OD}_{600}$ of 0.05 and grown $\mathrm{O} / \mathrm{N}$ (16h) at $28^{\circ} \mathrm{C}$ while shaking (200 rpm).

On day $3, \mathrm{O} / \mathrm{N}$ cultures were re-inoculated at $\mathrm{OD}_{600}$ of 0.2 in rich raffinose medium $(2 \%[\mathrm{w} / \mathrm{v}]$ Bacto peptone, $1 \%[\mathrm{w} / \mathrm{v}]$ Bacto yeast extract, $2 \%[\mathrm{w} / \mathrm{v}]$ raffinose, $0.1 \%[\mathrm{w} / \mathrm{v}]$ glucose) and grown to an $\mathrm{OD}_{600}$ of 0.5 (approx. 3-4h). An uninduced sample was taken before adding $2 \%$ galactose $[\mathrm{w} / \mathrm{v}]$ to the cultures in order to induce protein expression. At the desired time points, samples were taken and the absorbance measured at $600 \mathrm{~nm}$.

\section{Ubiquitination assay}

For the ubiquitination assay, cultures were treated with $75 \mu \mathrm{M}$ or $50 \mu \mathrm{M}$ MG132 (SelleckBio) or an equivalent volume of DMSO after 1 hour of galactose induction. Samples were taken at the indicated time points and total protein lysates were prepared.

\section{Degradation assay}

For the degradation assay, cultures were treated with 50 $\mu \mathrm{g} / \mathrm{ml}$ cycloheximide (CHX)(Sigma) after 1 hour of galactose induction. Samples were collected at the indicated time points in ice-cold sodium azide (10 $\mathrm{mM}$ final concentration) to prevent undesired protein degradation.

\section{Protein extracts and immunoblotting}

Protein extracts were prepared by breaking the cells with glass beads and acid precipitation essentially as previously described [39]. Aceton washed protein pellets were dried, re-

\section{REFERENCES}

1. Ciechanover A (2015) The unravelling of the ubiquitin system. Nat Rev Mol Cell Biol 16:322-324. doi: 10.1038/nrm3982

2. Swatek KN, Komander D (2016) Ubiquitin modifications. Cell Res 26:399-422. doi: 10.1038/cr.2016.39

3. Metzger MB, Hristova VA, Weissman AM (2012) HECT and RING finger families of E3 ubiquitin ligases at a glance. J Cell Sci 125:531537. doi: $10.1242 /$ jcs.091777

4. Buetow L, Huang DT (2016) Structural insights into the catalysis and regulation of E3 ubiquitin ligases. Nat Rev Mol Cell Biol 17:626-642. doi: $10.1038 / \mathrm{nrm} .2016 .91$

5. Erpapazoglou Z, Walker O, Haguenauer-Tsapis R (2014) Versatile roles of k63-linked ubiquitin chains in trafficking. Cells 3:1027-1088. doi: 10.3390/cells3041027 suspended in $1 \mathrm{X}$ Laemmli-sample buffer $(0.2 \mathrm{M}$ Tris- $\mathrm{HCl}[\mathrm{pH}$ 6.8], $1.5 \%$ sodium dodecyl sulfate [SDS], $10 \%$ glycerol, $1 \mathrm{mM}$ EDTA, $0.004 \%$ bromophenol blue) containing 50mM DTT, heated for $10 \mathrm{~min}$ at $65^{\circ} \mathrm{C}$ and separated by SDS-PAGE. Protein transfer and detection was done essentially as previously described [40]. The primary antibodies used included anti-HA rat monoclonal peroxidase-conjugated antibody (3F10; Roche), anti-Myc mouse monoclonal antibody (Cell Signaling CST2276), anti-myc-HRP (Thermo Fisher R951-25), anti-V5-HRP (Thermo Fisher R961-25), anti-Flag monoclonal antibody (M2 Sigma) and anti-Hexokinase polyclonal antibody.

\section{ACKNOWLEDGEMENTS}

We are grateful to Jens Nilsen for the the PTEF-URA plasmid, to Ype Elgersma for the mouse brain CDNA, to Jon Huibregtse for the \#95 plasmid, to Mike Ellison for the pES7 plasmid and to Stijn Bossuyt for his comments and suggestions. This research was supported by grants from the Netherlands Organization of Scientific Research (NWOZoN-MW; 91209046) and the Angelman Syndrome Alliance (ASA) to Ben Distel.

\section{SUPPLEMENTAL MATERIAL}

All supplemental data for this article are available online at www.microbialcell.com.

\section{CONFLICT OF INTEREST}

The authors declare no conflict of interest.

\section{COPYRIGHT}

(C) 2017 Avagliano Trezza et al. This is an open-access article released under the terms of the Creative Commons Attribution (CC BY) license, which allows the unrestricted use, distribution, and reproduction in any medium, provided the original author and source are acknowledged.

Please cite this article as: Rossella Avagliano Trezza, Janny van den Burg, Nico van den Oever and Ben Distel (2017). A versatile plasmid system for reconstitution and analysis of mammalian ubiquitination cascades in yeast. Microbial Cell 5(3): 150-157. doi: $10.15698 /$ mic2018.03.620

6. Yau R, Rape M (2016) The increasing complexity of the ubiquitin code. Nat Cell Biol 18:579-586. doi: 10.1038/ncb3358

7. Glickman MH, Ciechanover A (2002) The ubiquitin-proteasome proteolytic pathway: destruction for the sake of construction. Physiol $\operatorname{Rev}$ 82:373-428. doi: 10.1152/physrev.00027.2001

8. Wójcik C, DeMartino GN (2003) Intracellular localization of proteasomes. Int J Biochem Cell Biol 35:579-589. doi: 10.1016/S13572725(02)00380-1

9. Jastrab JB, Darwin KH (2015) Bacterial Proteasomes. Annu Rev Microbiol 69:109-127. doi: 10.1146/annurev-micro-091014-104201

10. Pearce MJ, Mintseris J, Ferreyra J, Gygi SP, Darwin KH (2008) Ubiquitin-like protein involved in the proteasome pathway of Mycobacterium tuberculosis. Science 322:1104-1107. 


\section{doi: 10.1126/science.1163885}

11. Markson G, Kiel C, Hyde R, Brown S, Charalabous P, Bremm A, Semple J, Woodsmith J, Duley S, Salehi-Ashtiani K, Vidal M, Komander D, Serrano L, Lehner P, Sanderson CM (2009) Analysis of the human E2 ubiquitin conjugating enzyme protein interaction network. Genome Res 19:1905-1911. doi: 10.1101/gr.093963.109

12. Li W, Bengtson $M H$, Ulbrich A, Matsuda A, Reddy VA, Orth A, Chanda SK, Batalov S, Joazeiro CA (2008) Genome-wide and functional annotation of human E3 ubiquitin ligases identifies MULAN, a mitochondrial E3 that regulates the organelle's dynamics and signaling. PLoS ONE 3:e1487. doi: 10.1371/journal.pone.0001487

13. Keren-Kaplan T, Attali I, Motamedchaboki K, Davis BA, Tanner N, Reshef $Y$, Laudon E, Kolot M, Levin-Kravets O, Kleifeld O, Glickman M, Horazdovsky BF, Wolf DA, Prag G (2011) Synthetic biology approach to reconstituting the ubiquitylation cascade in bacteria. EMBO J 31(2):378-90. doi: 10.1038/emboj.2011.397

14. Collins GA, Gomez TA, Deshaies RJ, Tansey WP (2010) Combined chemical and genetic approach to inhibit proteolysis by the proteasome. Yeast 27:965-974. doi: 10.1002/yea.1805

15. Kishino $T$, Lalande M, Wagstaff J (1997) UBE3A/E6-AP mutations cause Angelman syndrome. Nat Genet 15:70-73. doi: 10.1038/ng0197-70

16. Matsuura T, Sutcliffe JS, Fang P, Galjaard RJ, Jiang YH, Benton CS, Rommens JM, Beaudet AL (1997) De novo truncating mutations in E6AP ubiquitin-protein ligase gene (UBE3A) in Angelman syndrome. Nat Genet 15:74-77. doi: 10.1038/ng0197-74

17. Gietz RD, Sugino A (1988) New yeast-Escherichia coli shuttle vectors constructed with in vitro mutagenized yeast genes lacking sixbase pair restriction sites. Gene 74:527-534. doi: 10.1016/03781119(88)90185-0

18. West RW, Yocum RR, Ptashne M (1984) Saccharomyces cerevisiae GAL1-GAL10 divergent promoter region: location and function of the upstream activating sequence UASG. Molecular and Cellular Biology 4:2467-2478. doi: 10.1128/MCB.4.11.2467

19. Weinhandl K, Winkler M, Glieder A, Camattari A (2014) Carbon source dependent promoters in yeasts. Microb Cell Fact 13:5. doi: 10.1186/1475-2859-13-5

20. Cottrelle P, Thiele D, Price VL, Memet S, Micouin JY, Marck C, Buhler JM, Sentenac A, Fromageot $P$ (1985) Cloning, nucleotide sequence, and expression of one of two genes coding for yeast elongation factor 1 alpha. J Biol Chem 260:3090-3096. PMID: 2982849

21. Funk M, Niedenthal R, Mumberg D, Brinkmann K, Rönicke $V$, Henkel T (2002). Vector systems for heterologous expression of proteins in Saccharomyces cerevisiae. Meth Enzymol 350:248-257. doi: 10.1016/S0076-6879(02)50967-8

22. Mumberg D, Müller R, Funk M (1995). Yeast vectors for the controlled expression of heterologous proteins in different genetic backgrounds. Gene 156:119-122. doi: 10.1016/0378-1119(95)00037-7

23 Mumberg D, Müller R, Funk M (1994) Regulatable promoters of Saccharomyces cerevisiae: comparison of transcriptional activity and their use for heterologous expression. Nucleic Acids Res 22:57675768. doi: 10.1093/nar/22.25.5767

24. Macreadie IG, Jagadish MN, Azad AA, Vaughan PR (1989) Versatile cassettes designed for the copper inducible expression of proteins in yeast. Plasmid 21:147-150. doi: 10.1016/0147-619X(89)90059-0

25. Scheffner M, Huibregtse JM, Vierstra RD, Howley PM (1993) The HPV-16 E6 and E6-AP complex functions as a ubiquitin-protein ligase in the ubiquitination of p53. Cell 75:495-505. doi: 10.1016/0092-8674(93)90384-3

26. Lee $\mathrm{DH}$, Goldberg AL (1998) Proteasome inhibitors: valuable new tools for cell biologists. Trends Cell Biol 8:397-403. doi: 10.1016/S0962-8924(98)01346-4

27. Finley D, Ulrich HD, Sommer T, Kaiser $P$ (2012) The ubiquitinproteasome system of Saccharomyces cerevisiae. Genetics 192:319360. doi: 10.1534/genetics.112.140467

28. Flotho A, Melchior $F$ (2013) Sumoylation: a regulatory protein modification in health and disease. Annu Rev Biochem 82:357-385. doi: 10.1146/annurev-biochem-061909-093311

29. Lallemand-Breitenbach $V$, de Thé $H$ (2010) PML nuclear bodies. Cold Spring Harb Perspect Biol 2:a000661. doi: 10.1101/cshperspect.a000661

30. Kamitani T, Kito K, Nguyen HP, Wada H, Fukuda-Kamitani T, Yeh ET (1998) Identification of three major sentrinization sites in PML. J Biol Chem 273:26675-26682. doi: 10.1074/jbc.273.41.26675

31. Hay RT (2005) SUMO: a history of modification. Molecular Cell 18:1-12. doi: 10.1016/j.molcel.2005.03.012

32. Quimby BB, Yong-Gonzalez V, Anan T, Strunnikov AV, Dasso M (2006) The promyelocytic leukemia protein stimulates SUMO conjugation in yeast. Oncogene 25, 2999-3005. doi: 10.1038/sj.onc.1209335

33. Elgersma Y, van den Berg M, Tabak HF, Distel B (1993) An efficient positive selection procedure for the isolation of peroxisomal import and peroxisome assembly mutants of Saccharomyces cerevisiae. Genetics 135:731-740. PMID: 7507454

34. Wattanachaisaereekul S, Lantz AE, Nielsen ML, Nielsen J (2008) Production of the polyketide 6-MSA in yeast engineered for increased malonyl-CoA supply. Metab Eng 10:246-254. doi: 10.1016/j.ymben.2008.04.005

35. Williams C, van den Berg M, Panjikar S, Stanley WA, Distel B, Wilmanns $M$ (2012) Insights into ubiquitin-conjugating enzyme/ coactivator interactions from the structure of the Pex $4 p: P e x 22 p$ complex. EMBO J 31:391-402. doi: 10.1038/emboj.2011.411

36. Gueldener U, Heinisch J, Koehler GJ, Voss D, Hegemann JH (2002) A second set of loxP marker cassettes for Cre-mediated multiple gene knockouts in budding yeast. Nucleic Acids Res 30:e23. doi: 10.1093/nar/30.6.e23

37. Bajwa W, Torchia TE, Hopper JE (1988) Yeast regulatory gene GAL3: carbon regulation; UASGal elements in common with GAL1, GAL2, GAL7, GAL10, GAL80, and MEL1; encoded protein strikingly similar to yeast and Escherichia coli galactokinases. Mol Cellular Biol 8:3439-3447. doi: 10.1128/MCB.8.8.3439

38. Schiestl RH, Gietz RD (1989) High efficiency transformation of intact yeast cells using single stranded nucleic acids as a carrier. Curr Genet 16:339-346. doi: 10.1007/BF00340712

39. Elgersma $Y$, Kwast $L$, van den Berg $M$, Snyder WB, Distel $B$, Subramani S, Tabak HF (1997) Overexpression of Pex15p, a phosphorylated peroxisomal integral membrane protein required for peroxisome assembly in S.cerevisiae, causes proliferation of the endoplasmic reticulum membrane. EMBO J 16:7326-7341. doi 10.1093/emboj/16.24.7326

40. Gkourtsa A, van den Burg J, Strijbis K, Avula T, Bijvoets S, Timm D Hochstenbach F, Distel B (2015) Identification and characterization of Rvs162/Rvs167-3, a novel N-BAR heterodimer in the human fungal pathogen Candida albicans. Eukaryot Cell 14:182-193. doi: 10.1128/EC.00282-14 